\title{
BMJ Open Training approaches for the deployment of a mechanical chest compression device: a randomised controlled manikin study
}

\author{
Keith Couper, ${ }^{1,2}$ Rochelle M Velho, ${ }^{1,2}$ Tom Quinn, ${ }^{3}$ Anne Devrell, ${ }^{4}$ Ranjit Lall, ${ }^{1}$ \\ Barry Orriss, ${ }^{4}$ Joyce Yeung, ${ }^{1,2}$ Gavin D Perkins ${ }^{1,2}$
}

To cite: Couper K, Velho RM, Quinn T, et al. Training approaches for the deployment of a mechanical chest compression device: a randomised controlled manikin study. BMJ Open 2018;8:e019009. doi:10.1136/ bmjopen-2017-019009

- Prepublication history and additional material for this paper are available online. To view these files, please visit the journal online (http://dx.doi org/10.1136/bmjopen-2017019009).

Received 8 August 2017 Revised 15 November 2017 Accepted 19 December 2017

Check for updates

${ }^{1}$ Warwick Clinical Trials Unit, University of Warwick, Coventry, UK

${ }^{2}$ Academic Department of Anaesthesia, Critical Care, Pain and Resuscitation, Heart of England NHS Foundation Trust, Birmingham, UK

${ }^{3}$ Faculty of Health, Social Care and Education, Kingston University and St George's, University of London, London, UK

${ }^{4}$ Patient and Public representative, University of Warwick, Coventry, UK

Correspondence to

Dr Keith Couper

k.couper@warwick.ac.uk

\section{ABSTRACT}

Objectives To evaluate the effect of training strategy on team deployment of a mechanical chest compression device.

Design Randomised controlled manikin trial. Setting Large teaching hospital in the UK.

Participants Twenty teams, each comprising three clinicians. Participating individuals were health professionals with intermediate or advanced resuscitation training.

Interventions Teams were randomised in a 1:1 ratio to receive either standard mechanical chest compression device training or pit-crew device training. Training interventions lasted up to $1 \mathrm{~h}$. Performance was measured immediately after training in a standardised simulated cardiac arrest scenario in which teams were required to deploy a mechanical chest compression device.

Primary and secondary outcome measures Primary outcome was chest compression flow fraction in the minute preceding the first mechanical chest compression. Secondary outcomes included cardiopulmonary resuscitation quality and mechanical device deployment metrics, and non-technical skill performance. Outcomes were assessed using video recordings of the test scenario. Results In relation to the primary outcome of chest compression flow fraction in the minute preceding the first mechanical chest compression, we found that pitcrew training was not superior to standard training (0.76 ( $95 \% \mathrm{Cl} 0.73$ to 0.79 ) vs 0.77 (95\% $\mathrm{Cl} 0.73$ to 0.82 ), mean difference $-0.01(95 \% \mathrm{Cl}-0.06$ to 0.03$), \mathrm{P}=0.572)$. There was also no difference between groups in performance in relation to any secondary outcome.

Conclusions Pit-crew training, compared with standard training, did not improve team deployment of a mechanical chest device in a simulated cardiac arrest scenario.

Trial registration number ISRCTN43049287; Pre-results.

\section{BACKGROUND}

Mechanical chest compression devices provide a method to deliver high-quality chest compressions to patients in cardiac arrest. ${ }^{1}$ Despite evidence that manual chest compression delivery is often suboptimal and that high-quality chest compression
Strengths and limitations of this study

- This is the first randomised controlled study to investigate the effect of pit-crew training, compared with standard training, in the clinical area of cardiac arrest.

- This was a manikin study, such that it is unknown to what extent the findings can be reliably generalised to the clinical cardiac arrest setting.

- Outcomes were measured immediately after the training intervention, such that we did not investigate the long-term effect of each training intervention.

delivery is associated with improved survival, randomised controlled trials in out-of-hospital cardiac arrest (OHCA) have found that use of a mechanical chest compression device is not superior to manual chest compressions in relation to patient outcome..$^{2-5}$ In contrast, very low quality evidence from in-hospital cardiac arrest (IHCA) suggests that use of devices may be associated with improved patient outcome. $^{6}$

The key risk associated with use of mechanical chest compression devices is the pause in chest compressions associated with their deployment. ${ }^{1}$ In the LINC randomised controlled trial of the use of mechanical chest compression devices in OHCA, a substudy found that the median chest compression pause associated with device deployment was 36.0 s (IQR 19.5-45.5). ${ }^{7}$ Such pauses are associated with a reduction in coronary pressure during the early part of a cardiac arrest and may therefore offset the potential benefit of improved chest compression delivery associated with devices. ${ }^{8}$

Training as a team may be an effective strategy to optimise the delivery of care that is usually provided by a clinical team. ${ }^{9}$ Pit-crew resuscitation describes a concept where clinicians in a team act in a predetermined 
way to undertake set tasks in a specific order, akin to a formula one motor racing team. In a clinical before-after quality improvement study, the use of this concept alongside other interventions was associated with a marked reduction in the pause associated with device deployment in OHCA. ${ }^{10}$ However, the impact of the use of a pit-crew training approach has not, to date, been tested against other team training approaches in a randomised controlled trial.

\section{METHODS}

We conducted a randomised controlled parallel group trial to evaluate the impact of pit-crew training, compared with standard training, on mechanical chest compression deployment in a simulated cardiac arrest. The mechanical chest compression device used was the LUCAS-2 mechanical chest compression device (Jolife AB/Physio Control, Lund, Sweden). Outcomes were evaluated through a video-recorded simulation test that was undertaken immediately after the training intervention.

All participants provided written informed consent prior to receiving any study intervention.

\section{Participants}

Teams of three clinicians were recruited to participate in the trial. To be eligible to participate, individual clinicians were required to be registered health professionals with current Immediate Life Support or Advanced Life Support (ALS) certification, and to have completed the online manufacturer's device training package. Participants were not eligible if they had an injury or disability that prevented use or handling of the device, or if they had received practical training in the use of the device in the preceding 6 months.

Each team was required to be composed of three eligible clinicians, of which one was required to be an ALS-certificated provider or instructor. As such, teams were broadly reflective of the standard required of IHCA teams in the UK. ${ }^{11}$

\section{Study process}

We advertised the study at the hospital site through posters in staff areas, emails to staff and face-to-face discussions. Staff that consented to participate in the study were asked for their availability and allocated a training slot. As such, teams were created based on convenience, in that we established teams based on individual clinician's availability to attend training at a specified time. This broadly reflects how hospital cardiac arrest teams are created in clinical practice, in that the designated team will be drawn from clinicians who have been scheduled to work a particular shift.

On attendance at the training session, the team was randomised using a simple randomisation system provided by an internet-based randomisation service, which ensured allocation concealment (Sealed Envelope, London, UK). Teams were randomly allocated in a 1:1 ratio to receive either pit-crew training or standard training. After randomisation, a researcher $(\mathrm{KC})$, with experience in teaching ALS courses, immediately delivered the allocated training intervention. The team was not blinded to their allocated training intervention.

Following the training, the teams undertook a standardised manikin-based simulation test. The team acted as a hospital cardiac arrest team. The scenario, given in the SBAR format (situation, background, assessment, recommendation), described a 62-year-old male who had undergone tracheal intubation due to hypoxia secondary to a probable pulmonary embolus and who had subsequently become haemodynamically unstable leading to cardiac arrest. ${ }^{12}$ During the scenario, a mechanical chest compression device arrived during the second cardiopulmonary resuscitation (CPR) cycle, and the team was required to use the device. The length of the scenario was approximately $8 \mathrm{~min}$ (four cycles of CPR).

The simulation test was video-recorded. Two digital video recorders were used to mitigate against the risk of data loss and possible obstruction of a camera by participants.

\section{Study intervention}

An overview of the two training approaches, based on the TIDieR (template for intervention description and replication) framework, is included as an electronic supplement (online supplementary tables S1 and S2). ${ }^{13}$ Both training approaches incorporated a presentation on device deployment and use, followed by an opportunity for the team to practise these skills. Skill practice was scenario-based, with feedback following each scenario. It was anticipated that training would last approximately $45 \mathrm{~min}$, although teams were allowed to practice for as long as required.

The key difference between the training approaches was that teams randomised to pit-crew training received an overview of the concept and potential value of the pit-crew system in the training presentation, together with guidance on how to operationalise the pit-crew system in deploying the mechanical chest compression device. This pit-crew training system requires team members to adopt a nominated role that is associated with specific tasks, with team members trained to perform tasks in a coordinated manner. These concepts were also highlighted in feedback during practice scenarios.

In both training approaches, teams were trained to deploy the device in two stages, such that there was a pause for the deployment of the device back plate followed by the resumption of CPR and then a subsequent pause to enable deployment of the upper part of the mechanical device.

\section{Outcome measures}

The primary outcome was the chest compression flow fraction in the minute preceding the first mechanical chest compression. Flow fraction describes the proportion of time in which chest compressions are being delivered 


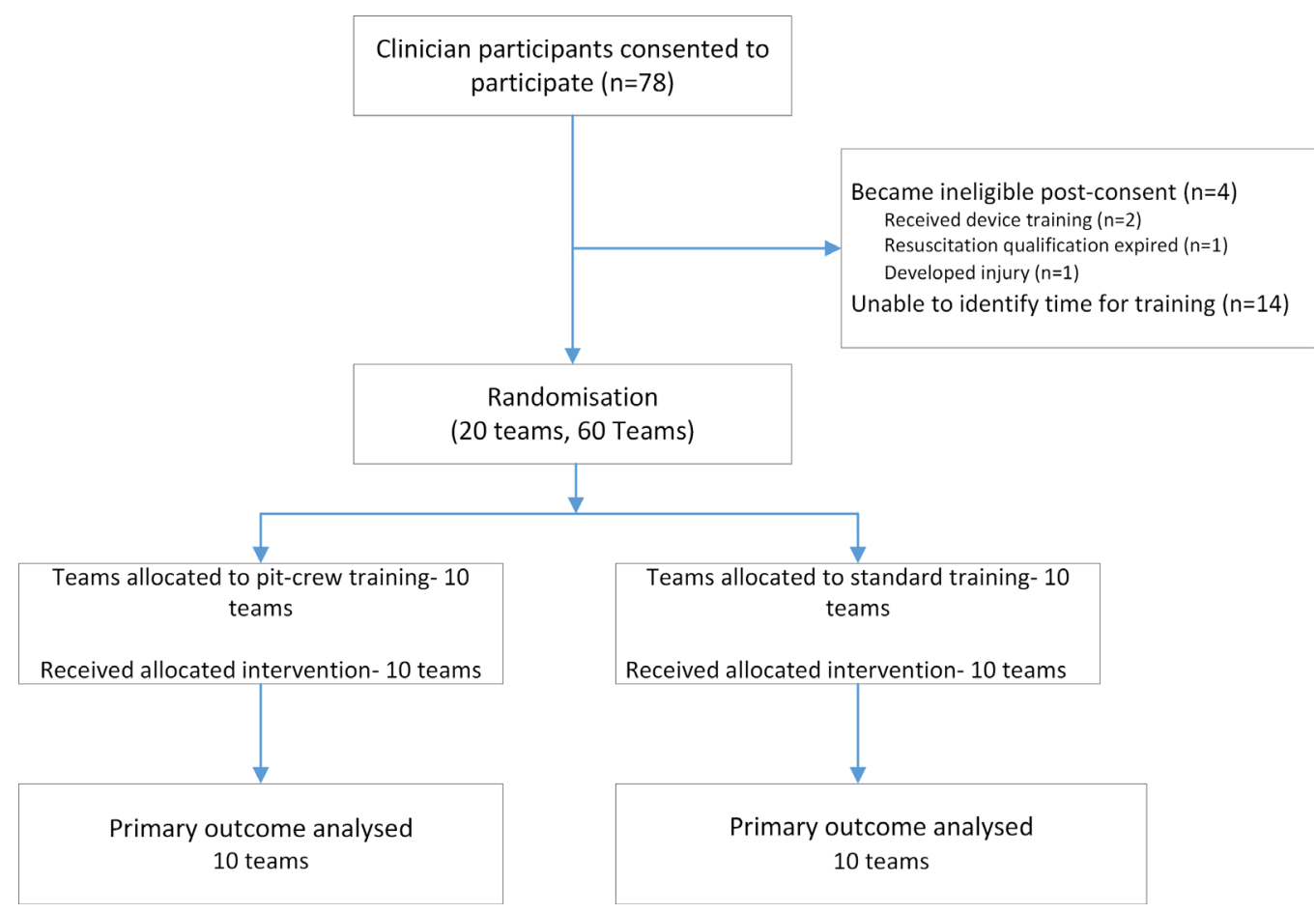

Figure 1 Study flow diagram.

over a designated period. This specific outcome was selected as it enabled us to capture all pauses that may be attributable to device deployment.

There were a number of secondary outcome measures including chest compression flow fraction (prior to the first mechanical chest compression, following the first

\begin{tabular}{|c|c|c|}
\hline & $\begin{array}{l}\text { Pit-crew } \\
\text { training } \\
\text { (10 teams) }\end{array}$ & $\begin{array}{l}\text { Standard training } \\
\text { (10 teams) }\end{array}$ \\
\hline \multicolumn{3}{|c|}{ Teams with $\mathrm{n}$ doctors, $\mathrm{n}(\%)$} \\
\hline 0 & $3(30)$ & $3(30)$ \\
\hline 1 & $5(50)$ & $2(20)$ \\
\hline 2 & $2(20)$ & $3(30)$ \\
\hline 3 & $0(0)$ & $2(20)$ \\
\hline \multicolumn{3}{|c|}{ Teams with n Advanced Life Support instructors, n (\%) } \\
\hline 0 & $4(40)$ & $5(50)$ \\
\hline 1 & $2(20)$ & $4(40)$ \\
\hline 2 & $3(30)$ & $1(10)$ \\
\hline 3 & $1(10)$ & $0(0)$ \\
\hline $\begin{array}{l}\text { Teams with at least } \\
\text { one member that has } \\
\text { previously received } \\
\text { device training, } \mathrm{n}(\%)\end{array}$ & $5(50)$ & $4(40)$ \\
\hline $\begin{array}{l}\text { Teams with at least } \\
\text { one member with } \\
\text { experience of using } \\
\text { a device in clinical } \\
\text { practice, } n(\%)\end{array}$ & $9(90)$ & $10(100)$ \\
\hline
\end{tabular}

mechanical chest compression, whole event), the duration of chest compression pauses associated with device deployment and non-technical skills measured using the Team Emergency Assessment Measure (TEAM) tool. ${ }^{14}$

Participating teams were not routinely informed as to the precise outcome measures being collected. However, a key focus of training interventions was the minimisation of pauses during deployment, and outcomes were recorded in the study entry on the trial registration website.

\section{Data management}

Videos were reviewed by two researchers (KC, RMV) independently. The first reviewer $(\mathrm{KC})$ delivered the training intervention and so was not blinded to training allocation. The second reviewer (RMV) was blinded to training allocation. Videos were viewed using software that enabled timings to be derived to the nearest one-tenth of a second.

We assessed agreement between video reviewers by computing the average difference and $95 \% \mathrm{CI}$ or median difference and IQR for each outcome and plotting data using a Bland-Altman plot. ${ }^{15}$ Bland-Altman plots visually depict the level of agreement between two reviewers, with good agreement represented by a small difference between the upper and lower limit of agreement and the majority of measurements falling between the limits of agreement. We used the mean value of the two reviewers in the analysis.

\section{Statistical analysis}

Our planned sample size of 20 teams (three clinicians per team) was based on demonstrating an absolute increase in flow fraction in the minute preceding the 
Table 2 Individual participant characteristics

\begin{tabular}{|c|c|c|}
\hline & Pit-crew training $(\mathrm{N}=30)$ & Standard training $(\mathrm{N}=30)$ \\
\hline \multicolumn{3}{|l|}{ Gender } \\
\hline Male, n (\%) & $12(40.0)$ & $14(46.7)$ \\
\hline \multicolumn{3}{|l|}{ Health profession, $\mathrm{n}(\%)$} \\
\hline Doctor & $9(30)$ & $14(46.7)$ \\
\hline Nurse & $21(70.0)$ & $12(40.0)$ \\
\hline Operating department practitioner & - & $1(3.3)$ \\
\hline Paramedic & - & $2(6.7)$ \\
\hline Other & - & $1(3.3)$ \\
\hline Professional experience, years, median (IQR) & $13.5(6.3-21.3)$ & $8.0(3.8-13.5)$ \\
\hline \multicolumn{3}{|l|}{ Grade, n (\%) } \\
\hline Band 5/FY1 & $3(10.0)$ & $5(16.7)$ \\
\hline Band 6/SHO & $8(26.7)$ & $11(36.7)$ \\
\hline Band 7/registrar & $14(46.7)$ & $9(30.0)$ \\
\hline Band 8+/consultant & $5(16.7)$ & $5(16.7)$ \\
\hline \multicolumn{3}{|l|}{ Current speciality, n (\%) } \\
\hline Acute medicine & $6(20.0)$ & $5(16.7)$ \\
\hline Critical care & $11(36.7)$ & $20(66.7)$ \\
\hline Emergency medicine & $6(20.0)$ & $2(6.7)$ \\
\hline Surgery & $3(10.0)$ & $2(6.7)$ \\
\hline Other & $4(13.3)$ & $1(3.3)$ \\
\hline \multicolumn{3}{|l|}{ Resuscitation qualification, n (\%) } \\
\hline Immediate Life Support provider & $7(23.3)$ & 7 (23.3) \\
\hline Advanced Life Support provider & $12(40.0)$ & $17(56.7)$ \\
\hline Advanced Life Support instructor & $11(36.7)$ & $6(20.0)$ \\
\hline $\begin{array}{l}\text { Approximate number of cardiac arrests attended in last } 6 \text { months, } \\
\text { median (IQR) }\end{array}$ & $4.5(2.0-10.0)$ & $8.0(1.8-15.8)$ \\
\hline Previously received device training, yes, $\mathrm{n}(\%)$ & $7(23.3)$ & $4(13.3)$ \\
\hline \multicolumn{3}{|l|}{ Previous use of a device in clinical practice, yes, $n(\%)$} \\
\hline If yes, which device, $\mathrm{n}(\%)$ & $18(60.0)$ & $17(56.7)$ \\
\hline LUCAS & $10(55.6)$ & $8(47.1)$ \\
\hline AutoPulse & $11(61.1)$ & $10(58.8)$ \\
\hline Thumper & $2(11.1)$ & $1(5.9)$ \\
\hline If yes, how many times used, median (IQR) & $2.5(1.0-5.8)$ & $3.0(1.5-4.5)$ \\
\hline
\end{tabular}

first mechanical chest compression of 0.15 (baseline $0.58, \mathrm{SD} 0.10)$ at $90 \%$ power with a $\mathrm{P}$ value of 0.05 . Due to the nature of the study, we did not increase the sample size to account for drop-outs. If a team member did not attend their allocated training session, then the team would not be eligible and so would not be randomised.

Data analysis was based on intention-to-treat principles. For baseline team and individual data, we report categorical variables as number and percentage, while continuous variables are described as mean $(95 \% \mathrm{CI})$ or median (IQR), depending on the normality of the data.

All outcomes are assessed at the team level. For normally distributed continuous outcomes, we summarise team performance as mean $(95 \% \mathrm{CI})$, and compare groups using an independent t-test and report the mean difference, $95 \% \mathrm{CI}$ and $\mathrm{P}$ value. For non-normally distributed continuous outcomes, we summarise group performance as median (IQR) and compare groups using a Mann-Whitney $\mathrm{U}$ test and report the $P$ value.

\section{RESULTS}

Between June 2016 and September 2016, 78 clinicians consented to participate in the study (figure 1). Of these, 60 participated in randomised teams. Four participants were excluded after giving consent to participate but prior to randomisation (one identified that their 
Table 3 Outcome measures (based on average from two videotape reviewers)

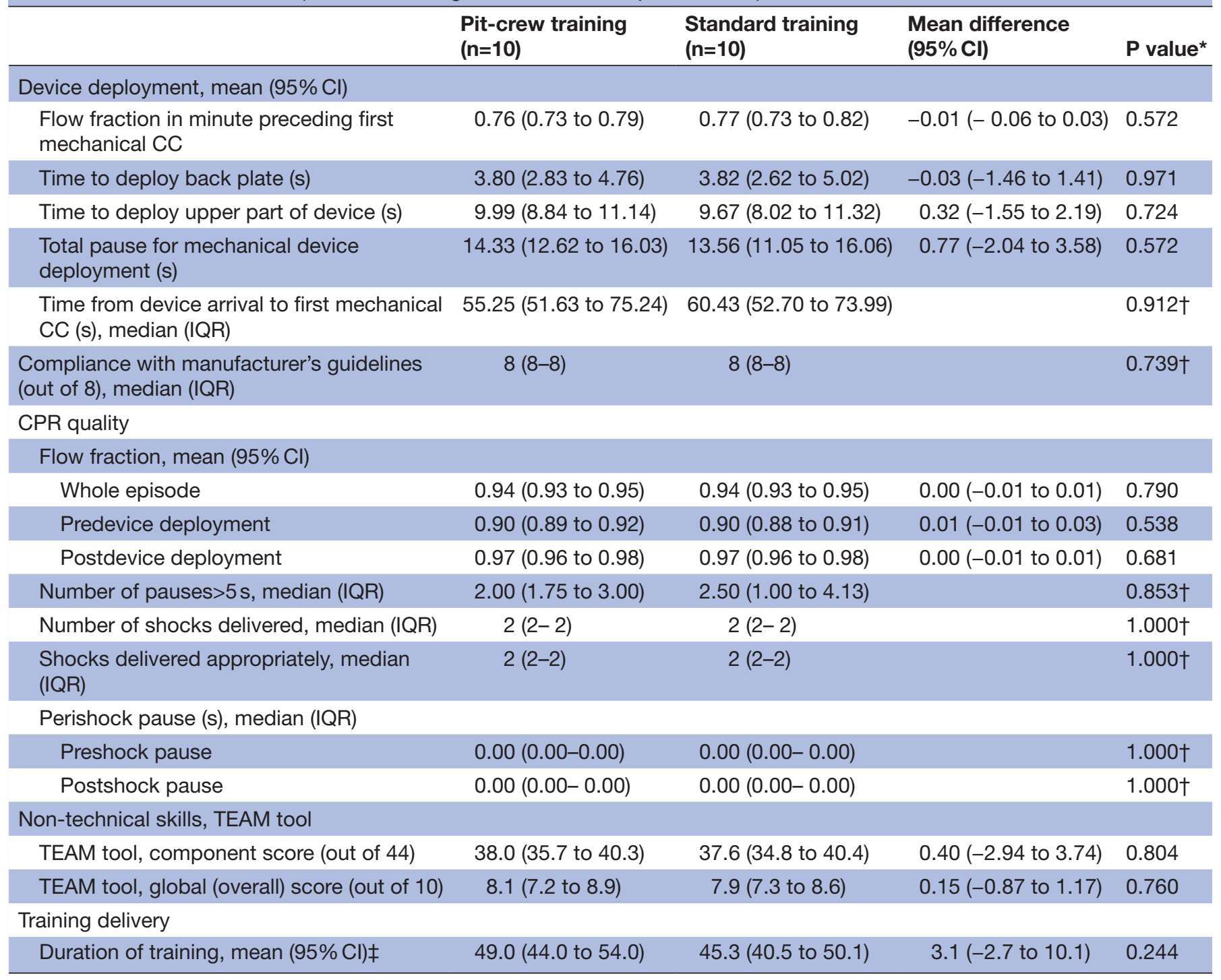

*By independent t-test unless stated.

†By Mann-Whitney U test.

‡Data point measured directly during training intervention, so not based on assessment from two reviewers.

$\mathrm{CC}$, chest compression; CPR, cardiopulmonary resuscitation; TEAM, Team Emergency Assessment Measure.

resuscitation certification had expired, one developed an injury that prevented use of the device and two received previous practical mechanical device training). For the remaining 13 participants, we were unable to identify a convenient time for training prior to randomising the 20 teams required.

Demographic data at the team and individual level are shown in table 1 and table 2, respectively. Team characteristics were comparable between groups. There were some differences between groups at the individual participant level in relation to, for example, clinical experience and specialty.

For the primary outcome, the average difference between reviewers for the 20 cases was 0.01 (95\% CI -0.01 to 0.02 ). Bland-Altman and average differences for other outcomes are included in the electronic supplement (online supplementary table S3 and figure S1). Based on these data, outcome analyses are based on the average data from the two reviewers.

In relation to the primary outcome, we found no difference in the flow fraction preceding the first mechanical chest compression between study groups $(0.76$ (95\% CI 0.73 to 0.79 ) vs 0.77 (95\% CI 0.73 to 0.82 ), mean difference -0.01 ( $95 \%$ CI -0.06 to 0.03$), \mathrm{P}=0.572$ ) (table 3$)$.

The chest compression pause associated with the deployment time of both the back plate and upper part of the device being similar between groups. We also observed no difference between groups in relation to any other secondary outcome.

There were no study adverse events, and all training interventions were delivered as planned, with time taken to deliver training interventions being similar between groups (table 3). During one simulation test (pit-crew training arm), the device battery failed as due to 
researcher error the device battery was not charged prior to training. This battery failure did not affect the primary outcome, but did marginally reduce flow fraction across the whole scenario and following device deployment.

\section{DISCUSSION}

In this randomised controlled manikin trial, we found that the use of a pit-crew training approach, compared with a standard training approach, did not affect the deployment of a mechanical chest compression device in simulated IHCA. Similarly, the use of pit-crew training did not affect any other device deployment, CPR quality or non-technical skills metric.

Our study, in contrast to previous pit-crew cardiac arrest studies, directly compared pit-crew training with a standard training approach. ${ }^{10}{ }^{16-20}$ While not directly comparable, it is noteworthy that previous studies have typically reported an association between implementation of pit-crew principles and improvements in process and patient-focussed outcomes. For example, in a before-after OHCA clinical study, the implementation of pit-crew training alongside real-time feedback and postevent debriefing was associated with an improvement in neurologically intact survival following OHCA (OR 2.3, $95 \%$ CI 1.3 to 4.0$).^{19}$

There are four possible explanations for this apparent contrast in findings. First, previous studies have tended to implement pit-crew training alongside other interventions, such as real-time feedback, additional training and cardiac arrest debriefing. As such, the findings of previous studies may reflect the impact of the overall package of interventions, such that pit-crew training either did not have an independent effect or complementary interventions enhanced its effect. Second, previous studies have adopted an observational design, such that the findings may be attributable to unmeasured confounders rather than the intervention that was implemented.

A third explanation is that where team performance is high, as observed in this study, pit-crew training provides no additional benefit. In Levy et al's beforeafter study, where the pit-crew training intervention was associated with a marked improvement in care delivery, the median preintervention pause between the final manual chest compression and first mechanical chest compression prior to implementation of pit-crew training was $21 \mathrm{~s}$ (IQR 15 to 31 ).$^{10}$ In contrast, the mean total pause associated with device deployment in the standard training group in this study was about $14 \mathrm{~s}$, such that the opportunity for pit-crew training to have a measurable effect on team performance may have been limited.

Finally, it may be that pit-crew concepts provide more value in the clinical setting, compared with simulated cardiac arrest conditions. Replicating the reallife complexity of cardiac arrest management in the simulation laboratory is challenging. ${ }^{21}{ }^{22}$ It is possible that pit-crew training provides most advantage in the unpredictable clinical setting, where the system provides a clear framework to direct effective team functioning.

Our study has several weakness. First, we tested performance in a simulated cardiac arrest scenario that occurred immediately after the training intervention, such that we do not know how training interventions affected performance either in the longer term or in the clinical setting. Second, participants were often experienced clinicians with frequent exposure to cardiac arrest, so it is unclear whether findings are generalisable to less-experienced clinicians. Third, we found that performance in both groups was markedly better than that estimated in our power calculation, so our study was underpowered to reliably detect a clinically important difference between groups. Fourth, we did not capture participant views on the impact of training approaches on team functioning. Such information may have captured the personal learning style of individuals within the team and how receptive they were to the different training approaches. Finally, while agreement between video reviewers was generally good, there was some variation in relation to the TEAM tool outcomes. However, analysis at an individual reviewer level produced similar results to our overall planned analysis (data not presented).

\section{CONCLUSION}

In this randomised controlled manikin trial, we found that pit-crew training, compared with standard device training, did not improve the deployment of mechanical chest compression devices in a simulated cardiac arrest. The time taken to deliver training was similar. Future controlled trials should examine the effect of pit-crew training in the clinical setting and on longterm performance.

Acknowledgements We are grateful to the research participants for giving up their time to participate in the study. We gratefully acknowledge the support of Sister Julia Sampson (Heart of England NHS Foundation Trust) for her support in recruiting participants to the study.

Contributors KC conceived and designed the study, acquired the data, analysed the data, contributed to interpretation of the data, critically revised the manuscript for important intellectual content, drafted the manuscript and approved the final manuscript. RMV analysed the data, contributed to interpretation of the data, critically revised the manuscript for important intellectual content and approved the final manuscript. TQ conceived and designed the study, contributed to interpretation of the data, critically revised the manuscript for important intellectual content and approved the final manuscript. $A D$ conceived and designed the study, contributed to interpretation of the data, critically revised the manuscript for important intellectual content and approved the final manuscript. RL conceived and designed the study, contributed to interpretation of the data, critically revised the manuscript for important intellectual content and approved the final manuscript. B0 conceived and designed the study, contributed to interpretation of the data, critically revised the manuscript for important intellectual content and approved the final manuscript. JY contributed to interpretation of the data, critically revised the manuscript for important intellectual content and approved the final manuscript. GDP conceived and designed the study, contributed to interpretation of the data, critically revised the manuscript for important intellectual content and approved the final manuscript.

Funding KC and JY are supported as NIHR postdoctoral research fellows. GDP is an NIHR senior investigator. This research was funded by an NIHR Postdoctoral Research Fellowship (PDF 2015-08-109). 
Disclaimer The views expressed are those of the authors and not necessarily those of the NHS, the NIHR or the Department of Health. The funder had no role in the design of the study, data collection, data analysis and interpretation or the writing of the manuscript.

Competing interests $\mathrm{KC}, \mathrm{TQ}, \mathrm{RL}, \mathrm{JY}$ and GDP report that their organisations have received funding from NIHR for clinical trials on the use of mechanical chest compression devices.

Patient consent Obtained.

Ethics approval The study was reviewed and approved by the University of Warwick Biomedical and Scientific Research Ethics Committee.

Provenance and peer review Not commissioned; externally peer reviewed.

Data sharing statement The datasets generated and analysed during the current study are not publicly available to maintain participant confidentiality but are available from the corresponding author on reasonable request.

Open Access This is an Open Access article distributed in accordance with the Creative Commons Attribution Non Commercial (CC BY-NC 4.0) license, which permits others to distribute, remix, adapt, build upon this work non-commercially, and license their derivative works on different terms, provided the original work is properly cited and the use is non-commercial. See: http://creativecommons.org/ licenses/by-nc/4.0/

(C) Article author(s) (or their employer(s) unless otherwise stated in the text of the article) 2018. All rights reserved. No commercial use is permitted unless otherwise expressly granted.

\section{REFERENCES}

1. Couper K, Smyth M, Perkins GD. Mechanical devices for chest compression: to use or not to use? Curr Opin Crit Care 2015;21:188-94.

2. Wik L, Kramer-Johansen J, Myklebust H, et al. Quality of cardiopulmonary resuscitation during out-of-hospital cardiac arrest. JAMA 2005;293:299-304.

3. Gates S, Quinn T, Deakin CD, et al. Mechanical chest compression for out of hospital cardiac arrest: systematic review and metaanalysis. Resuscitation 2015;94:91-7.

4. Perkins GD, Lall R, Quinn T, et al. Mechanical versus manual chest compression for out-of-hospital cardiac arrest (PARAMEDIC): a pragmatic, cluster randomised controlled trial. Lancet 2015;385:947-55.

5. Talikowska M, Tohira $\mathrm{H}$, Finn J. Cardiopulmonary resuscitation quality and patient survival outcome in cardiac arrest: A systematic review and meta-analysis. Resuscitation 2015;96:66-77.

6. Couper K, Yeung J, Nicholson T, et al. Mechanical chest compression devices at in-hospital cardiac arrest: a systematic review and meta-analysis. Resuscitation 2016;103:24-31.
7. Esibov A, Banville I, Chapman FW, et al. Mechanical chest compressions improved aspects of CPR in the LINC trial. Resuscitation 2015;91:116-21.

8. Berg RA, Sanders AB, Kern KB, et al. Adverse hemodynamic effects of interrupting chest compressions for rescue breathing during cardiopulmonary resuscitation for ventricular fibrillation cardiac arrest. Circulation 2001;104:2465-70.

9. Chakraborti C, Boonyasai RT, Wright SM, et al. A systematic review of teamwork training interventions in medical student and resident education. J Gen Intern Med 2008;23:846-53.

10. Levy M, Yost D, Walker RG, et al. A quality improvement initiative to optimize use of a mechanical chest compression device within a high-performance CPR approach to out-of-hospital cardiac arrest resuscitation. Resuscitation 2015;92:32-7.

11. Resuscitation Council (UK). Quality standards for cardiopulmonary resuscitation practice and training. http://www.resus.org.uk/pages/ QSCPR_Acute.htm (accessed 24 May 2017).

12. Haig KM, Sutton S, Whittington J. SBAR: a shared mental model for improving communication between clinicians. Jt Comm J Qual Patient Saf 2006;32:167-75.

13. Hoffmann TC, Glasziou PP, Boutron I, et al. Better reporting of interventions: template for intervention description and replication (TIDieR) checklist and guide. BMJ 2014;348:g1687.

14. Cooper S, Cant R, Porter J, et al. Rating medical emergency teamwork performance: development of the Team Emergency Assessment Measure (TEAM). Resuscitation 2010;81:446-52.

15. Bland JM, Altman DG. Statistical methods for assessing agreement between two methods of clinical measurement. Lancet 1986;1:307-10.

16. Spiro JR, White S, Quinn N, et al. Automated cardiopulmonary resuscitation using a load-distributing band external cardiac support device for in-hospital cardiac arrest: a single centre experience of AutoPulse-CPR. Int J Cardiol 2015;180:7-14.

17. Bobrow BJ, Vadeboncoeur TF, Stolz U, et al. The influence of scenario-based training and real-time audiovisual feedback on outof-hospital cardiopulmonary resuscitation quality and survival from out-of-hospital cardiac arrest. Ann Emerg Med 2013;62:47-56.

18. Braithwaite S, Friesen JE, Hadley S, et al. A tale of three successful EMS systems. How coordinated "pit crew" procedures have helped improve cardiac arrest resuscitations in the field. JEMS 2014;Suppl:28-35.

19. Hopkins CL, Burk C, Moser S, et al. Implementation of pit crew approach and cardiopulmonary resuscitation metrics for out-ofhospital cardiac arrest improves patient survival and neurological outcome. J Am Heart Assoc 2016;5:e002892.

20. Ong ME, Quah JL, Annathurai A, et al. Improving the quality of cardiopulmonary resuscitation by training dedicated cardiac arrest teams incorporating a mechanical load-distributing device at the emergency department. Resuscitation 2013;84:508-14.

21. Perkins GD. Simulation in resuscitation training. Resuscitation 2007;73:202-11.

22. Yeung J. Transforming a team of experts into an expert team. Resuscitation 2016;101:A1-A2. 